University of Nebraska - Lincoln

DigitalCommons@University of Nebraska - Lincoln

Faculty Publications from the Department of Electrical \& Computer Engineering, Department Electrical and Computer Engineering

7-1-1993

Photoellipsometry determination of surface Fermi level in GaAs (100)

\author{
Yi-Ming Xiong \\ University of Nebraska-Lincoln \\ Paul G. Snyder \\ University of Nebraska-Lincoln, psnyder1@unl.edu \\ John A. Woollam \\ University of Nebraska-Lincoln, jwoollam1@unl.edu
}

Follow this and additional works at: https://digitalcommons.unl.edu/electricalengineeringfacpub

Part of the Electrical and Computer Engineering Commons

Xiong, Yi-Ming; Snyder, Paul G.; and Woollam, John A., "Photoellipsometry determination of surface Fermi level in GaAs (100)" (1993). Faculty Publications from the Department of Electrical and Computer Engineering. 71.

https://digitalcommons.unl.edu/electricalengineeringfacpub/71

This Article is brought to you for free and open access by the Electrical \& Computer Engineering, Department of at DigitalCommons@University of Nebraska - Lincoln. It has been accepted for inclusion in Faculty Publications from the Department of Electrical and Computer Engineering by an authorized administrator of DigitalCommons@University of Nebraska - Lincoln. 


\title{
Photoellipsometry determination of surface Fermi level in GaAs (100)
}

\author{
Yi-Ming Xiong \\ Department of Physics, Center for Microelectronic and Optical Materials Research. \\ University of Nebraska, Lincoln, Nebraska 68588
}

Paul G. Snyder and John A. Woollam

Department of Electrical Engineering, Center for Microelectronic and Optical Materials Research, University of Nebraska, Lincoln. Nebraska 68588

(Received 8 December 1992; accepted 14 March 1993)

\begin{abstract}
Photoellipsometry, a new contactless optical method, is presented. Related to photoreflectance, this method utilizes spectroscopic ellipsometry with the addition of a laser pump beam directed at nearly normal incidence onto the sample surface, where photoexcited electron-hole pairs modify the built-in slectric field as a result of the photovoltaic effect. The field-induced changes in the real and imaginary parts of the complex pseudodielectric function can be directly measured, and analyzed in terms of the pump beam power density or the probe beam photon energy to deternine parameters, such as surface Fermi level, bult-in field strength, depietion depth, broadening, criticai point energies, etc., of a semiconductor under study. Demonstration of this method was made with two n-type GaAs (100) substrates, where the measurements were taken in room air near the band-gap $E_{0}$ region, for determination of surface Fermi level.
\end{abstract}

\section{INTRODUCTION}

Because of its derivative nature (which produces sharp, highly structured spectra) and high sensitivity to surface and interface electric fields, electroreflectance ${ }^{1}$ (ER) is a useful technique for studying the band structure of semiconductors, and characterizing electric field-induced effects on these materials. ER measures electric field-induced changes in the reflectance spectra from a sample suriace, where electromodulation is accomplished by applying an alternating electric field. Commonly used configurations for producing the modulating field include immersing the sample in an electrolytic field or coating the sample with a transparent or semitransparent metallic film which serves as an electrode. Each of these configurations is likely to introduce perturbations to the sample surface, making the measured spectra difficult to interpret. A contactless form of $E R$ is photoreflectance ${ }^{2}$ ( $P R$ ), in which electromodulation is produced internally by photoexcited electron-hole pairs (free carriers) generated by a secondary light pump source. This method has been widely used as a tool for characterizing semiconductor band structure, surface Fermi level, built-in electric field strength, etc. ${ }^{2-6} \mathrm{Com}$ pared to ER, $P R$ is more powerful in that it is a contactless method, and therefore is noninvasive to the sample surface. In addition, this feature permits an independent control of the ambient and of the temperature, and has already proved useful for in sizu studies of semiconductors and semiconductor microstructures. ${ }^{7}$ In spite of these advantages, there are still some drawbacks; for example, both methods only measure the electric field-modulated reflectance spectra, and therefore are not overly sensitive to sample surface conditions and interference effects in layered structures.

Spectroscopic ellipsometry (SE), a contactless optical method, offers complementary features to ER and PR. Since its inception, ${ }^{8,9} \mathrm{SE}$ has been generally recognized as one of the most sensitive and reliable tools for accurate determination of the optical properties of a wide variety of bulk semiconductor materials, ${ }^{10-14}$ and for quantitative characterization of semiconductor surfaces and interfaces, ${ }^{15-18}$ as well as semiconductor multilayer structures. ${ }^{19-22} \mathrm{SE}$ measures the polarization state of a reflected light beam, rather than its intensity. Compared to ER and PR, SE has the following advantages: (1) high (monolayer) sensitivity to surface overlayers and other thin films; (2) direct measurement of both the real and imaginary parts of the bulk dielectric function $\epsilon$ without the need for a Kramers-Kronig transformation; (3) very high accuracy for $\epsilon$ measurements over a wide spectral domain; (4) nondestructive depth profiling capability through measurements as a function of photon energy. One drawback of $\mathrm{SE}$ is that it only measures the absolute $\varepsilon$ spectra, as opposed to the derivativelike spectra by the ER and PR methods. As a result, weaker structures, such as those by electric field-induced effects, are difficult to resolve, without being enhanced by numerical differentiation and smoothing techniques.

Modulation ellipsometry (ME) was first developed by Buckman and Bashara ${ }^{23}$ for analyzing electric fieldinduced effects on the dielectric functions of $\mathrm{Au}$ and $\mathrm{Ag}$. This method utilizes $S E$ with $E R$, therefore combining the features of both methods and providing more complete information not available in separate applications. Following similar experimental procedures by Buckman and Bashara, ${ }^{23}$ Tachi $e t$ al. ${ }^{24}$ and Glembocki ${ }^{25}$ studied the fieldinduced effects on the dielectric functions of $\mathrm{GaAs}$ and $\mathrm{Si}$, respectively. These results indicated that $M E$ indeed was able to provide more information about the sample from a single experiment; for instance, using $M E$, the fieldinduced changes in both the real and imaginary parts of $\epsilon$ can be simultaneously determined without recourse to the Kramers-Kronig analysis. Despite this and other advantages, ME still has not been wideiy used as an effective tool for characterizing semiconductors and other materials. 
One of the reasons is the difficulty in implementation of the technique itself; for example, phase-sensitive detection (lock-in amplifier) must be implemented in signal processing to extract the field-induced small changes in the measured spectra. Another major drawback is closely related to the contact configuration, in which the modulating field is applied. As in the case of ER, regardless of which contact method is chosen, surface perturbations are likely to occur, making the resulting spectra more difficult to interpret.

Photoellipsometry (PE) is a new contactless form of $M E$ and can be used to study the built-in electric fieldinduced effects on the dielectric functions of semiconductors. This method was developed based on the observation ${ }^{26}$ of the built-in field-induced FranzKeldysh effect ${ }^{27}$ on conventional SE spectra from GaAs/Al $\mathrm{Ga}_{1-x} \mathrm{As}$ heterostructures. PE incorporates conventional SE with the addition of a secondary laser pump beam directed at nearly normal incidence onto the sample surface. It thus combines the features of both $\mathrm{SE}$ and PR, and therefore is completely contactless and can be applied in any transparent ambient environment at any temperature. As in $P R$, the absorption of pump beam photons results in the creation of free carriers, which in turn modify the built-in field strength within the sample as a result of the photovoltaic effect. ${ }^{28}$ The field-induced change in the complex pseudodielectric function $\langle\epsilon\rangle$ can be directly measured using PE and analyzed with an appropriate method to determine parameters, such as surface Fermi level, built-in field strength, depletion depth, broadening, critical point (CP) energies, etc., of a given semiconductor. The pseudodielectric function $\langle\epsilon\rangle$ also includes all surface and depth-dependent effects possibiy present on $\epsilon$, such as surface native oxide overlayer and dielectric inhomogeneity. A major advantage of $\mathrm{PE}$ over reflectance modulation methods is that it allows direct measurements of fieldinduced changes in both the real and imaginary parts of $\langle\epsilon\rangle$, i.e., $\delta\left\langle\epsilon_{1}\right\rangle$ and $\delta\left\langle\epsilon_{2}\right\rangle$, on a wavelength-by-wavelength basis, without the need for a Kramers-Kronig transformation or electrical contacts to the sample. This unique feature permits a direct observation of the Franz-Keldysh effect from a given semiconductor. Moreover, because both $\delta\left\langle\epsilon_{1}\right\rangle$ and $\delta\left\langle\epsilon_{2}\right\rangle$ spectra are available experimentally, tighter constraints can be imposed on the models representing more complicated structures, thereby increasing the confidence in the calculated results.

The main objective in this work is to describe how $\mathrm{PE}$ can be used to determine the surface Fermi level, and the built-in surface electric feid strength, in bulk doped GaAs. To this end, two commercial $n$-type GaAs substrates were siudied in as-received conditions. The measured data were analyzed in terms of the pump beam power density and the probe beam photon energy.

\section{SAMPLES AND EXPERIMENT}

The two samples were commercial Si-doped $n$-type GaAs (100) wafer substrates, with nominal doping densities $N_{D} \approx 1.3 \times 10^{17}$ and $4.0 \times 10^{17} \mathrm{~cm}^{-3}$, respectively. The wafers were cut from single crystal GaAs ingots, grown by

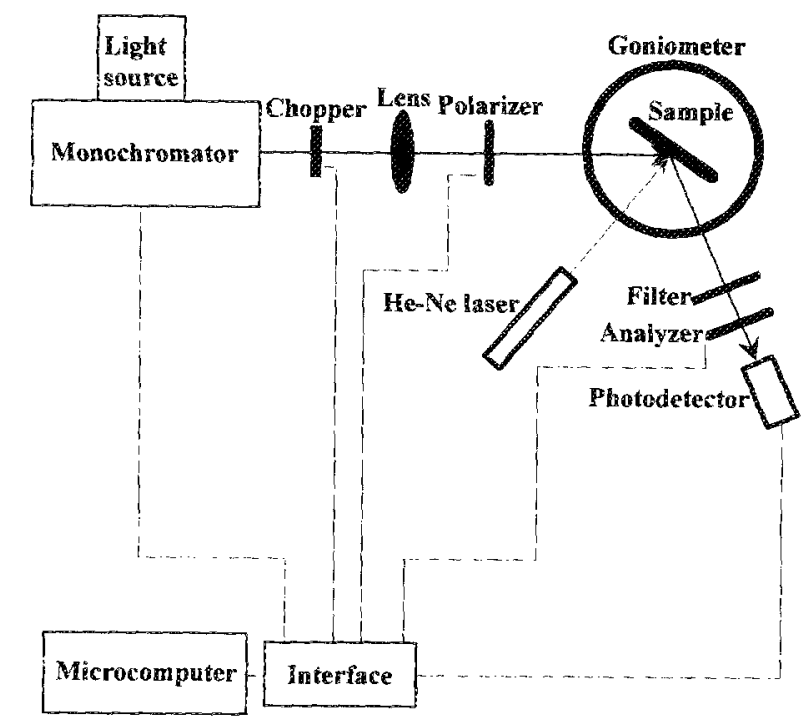

FIc. 1. Schematic diagram of the photoellipsometer apparatus.

the Bridgman method. The front surface of each sample was chemomechanically polished, giving a mirrorlike finish, and the back surface was unpolished.

Figure 1 shows the schematic diagram of the PE apparatus used. It consisted of a conventional rotating analyzer spectroscopic ellipsometer similar to the one described in Ref. 9, and a $7 \mathrm{~mW}$ He-Ne (with wavelength $\lambda=632.8$ $\mathrm{nm}$ ) laser used as the pump light source. The probe beam light source for the ellipsometer was a $75 \mathrm{~W}$ xenon lamp, coupled to a scanning monochromator with an average bandwidth of $\sim 2 \mathrm{~nm}$. The probe beam diameter was about $1 \mathrm{~mm}$. The laser pump beam, directed at nearly normal incidence onto the sample surface, was spatially filtered and expanded to completely overlap the probe beam sampled area. The pump beam intensity was controlled by a set of neutral density filters. Colored glass filter was used to prevent the scattered pump beam light from entering the $\mathrm{Si}$ detector.

The sampies were studied in as-received conditions. The measurements were first taken with two different pump beam power densities at a particular photon energy in the above-band-gap $E_{0}$ region, where the field-induced change in $\langle\epsilon\rangle$ was large. The measurements were then made with a single pump power density, in a spectral range from 1.30 to $1.65 \mathrm{eV}$. All the measurements were taken in air at $T=297 \mathrm{~K}$. The probe beam angle of incidence was $75^{\circ}$, near the pseudo-Brewster angle ${ }^{29}$ for GaAs. The average uncertainty for the eilipsometric parameters $\psi$ and $\Delta$ was within $\pm 0.005^{\circ}$ and $\pm 0.01^{\circ}$, respectively, in the above spectral range.

\section{SPECTROSCOPIC ELLIPSOMETRY AND PHOTOELLIPSOMETRY BASICS}

Details about ellipsometry can be found in Ref. 29 , only a brief description is given here. Eilipsometry deals with the measurements and interpretation of changes in the polarization state of a linearly polarized light undergoing ob- 
lique reffection from a sampie surface. In SE, one measures $\psi$ and $\Delta$, as a function of photon energy (or wavelength), which are defined by ${ }^{29}$

$$
\tan \psi \exp (i \Delta)=R_{p} / R_{s},
$$

where $R_{p}$ and $R_{s}$ are, respectively, the complex reflection coefficients for light polarized parallel $(p)$ and perpendicvlar $(s)$ to the plane of incidence. Both $R_{p}$ and $R_{s}$ contain information about the sample's optical properties.

With each pair of $\psi$ and $\Delta$ measured, the corresponding real and imaginary parts of the complex pseudodielectric function $\langle\epsilon\rangle$, i.e., $\left\langle\epsilon_{1}\right\rangle$ and $\left\langle\epsilon_{2}\right\rangle$, can be directly obtained, assuming a two-phase (ambient-substrate) model ${ }^{29}$

$$
\left\langle\epsilon_{1}\right\rangle=\epsilon_{a} \sin ^{2} \phi\left[1+\tan ^{2} \phi\left(\frac{\cos ^{2} 2 \psi-\sin ^{2} 2 \psi \sin ^{2} \Delta}{(1+\sin 2 \psi \cos \Delta)^{2}}\right)\right]
$$

and

$$
\left\langle\epsilon_{2}\right\rangle=\epsilon_{a} \sin ^{2} \phi \tan ^{2} \phi\left(\frac{\sin 4 \psi \sin \Delta}{(1+\sin 2 \psi \cos \Delta)^{2}}\right),
$$

where $\phi$ is the probe beam angle of incidence and $\epsilon_{a}$ is the dielectric constant of the ambient ( $\epsilon_{a} \approx 1$ in air). The term "pseudo" means that a simple uniform bulk substrate with no overlayers is assumed. Therefore, the effects of surface overlayers and other inhomogeneities, which are in reality present, are implicitly included in the $\langle\epsilon\rangle$.

For absorbing materials such as GaAs, SE has maximum sensitivity at the pseudo-Brewster condition, ${ }^{29}$ in which the reflection coeficient $R_{p}$ takes a minimum value. Noting that $R_{p}$ depends on the angle of incidence $\phi$ and the pseudodielectric function $\langle\epsilon\rangle$, the pseudo-Brewster condition is satisfied only for a particular angle $\phi_{B}$ (pseudoBrewster angle) and a particular value of $\langle\epsilon\rangle$ for a given sample under study. For GaAs and its alloy materials such as $\mathrm{Al}_{x} \mathrm{Ga}_{1}{ }_{x} \mathrm{As}, \phi_{B}$ is close to $75^{\circ} .14,18-21$ At $\phi_{B}$, an abrupt variation occurs, between -1 to 1 in the $\cos \Delta$ spectrum near a certain photon energy corresponding to a particular value of $\langle\epsilon\rangle$. This variation is extremely sensitive to any perturbations introduced in the sample, such as the presence of surface overiayers, and is responsibie for the high sensitivity of SE measurements.

$\mathrm{PE}$ incorporates $\mathrm{SE}$ with the addition of a secondary laser pump beam, directed at nearly normal incidence onto the sample surface. It directly measures the built-in fieldinduced changes in the real and imaginary parts of $\langle\epsilon\rangle$ for a given sample under study. $P E$ data are obtained by taking the difference between two sets of SE data $\langle\epsilon\rangle$, measured at the same photon energy without and with the pump beam on. Specifically, if $F_{1}$ and $F_{2}$ are the built-in electric fields in the direction normal to the surface, corresponding to the pump beam on and off state, respectively, the field-induced changes $\delta\left\langle\epsilon_{1}\right\rangle$ and $\delta\left\langle\epsilon_{2}\right\rangle$ are obtained using

$$
\begin{aligned}
\delta\left\langle\epsilon_{1}\left(F_{2}, F_{1}, E\right)\right\rangle= & \left\langle\epsilon_{1}(\text { pump beam off, } E)\right\rangle \\
& -\left\langle\epsilon_{1}(\text { pump beam on, } E)\right\rangle \\
\equiv & \left\{\epsilon_{1}\left(F_{2}, E\right)\right\rangle-\left\langle\epsilon_{1}\left(F_{1}, E\right)\right\rangle
\end{aligned}
$$

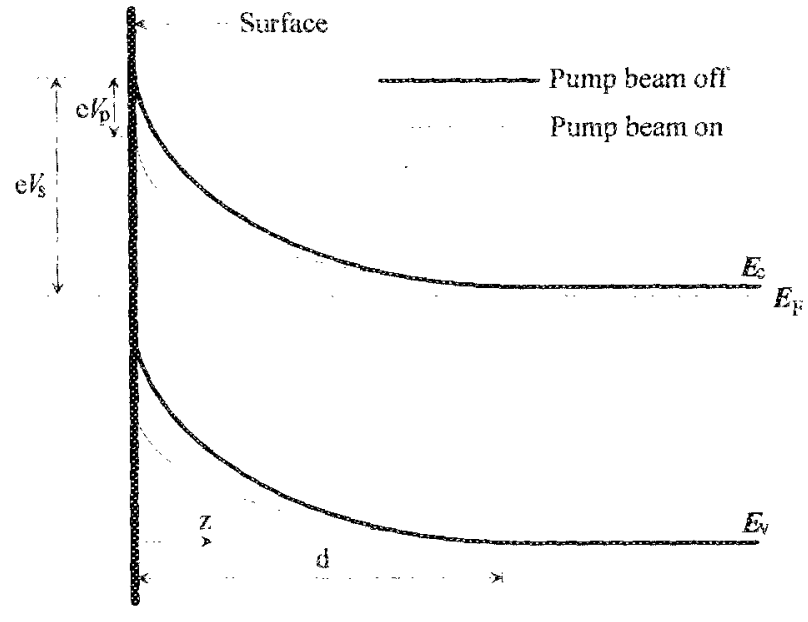

FKG. 2. Energy band diagram of an n-type GaAssurface with and without the effects of pump light irradiation.

and

$$
\begin{aligned}
\delta\left\langle\epsilon_{2}\left(F_{2}, F_{\xi}, E\right)\right\rangle= & \left\langle\epsilon_{2}(\text { pump beam off, } E)\right\rangle \\
& -\left\langle\epsilon_{2}(\text { pump beam on, } E)\right\rangle \\
\equiv & \left\langle\epsilon_{2}\left(F_{2}, E\right)\right\rangle-\left\langle\epsilon_{2}\left(F_{1}, E\right)\right\rangle,
\end{aligned}
$$

where $E$ represents the photon energy of the probe light, and $\left\langle\epsilon_{1}\left(F_{1}, E\right)\right\rangle,\left\langle\epsilon_{2}\left(F_{1}, E\right)\right\rangle,\left\langle\epsilon_{1}\left(F_{2}, E\right)\right\rangle$, and $\left\langle\epsilon_{2}\left(F_{2}, E\right)\right\rangle$ are obtained from Eqs. (2a) and $(2 b)$. These two equations are the means by which $\mathrm{PE}$ data are obtained. Equations (3a) and (3b) can be also writter, respectively, as

$$
\begin{aligned}
\delta\left\langle\epsilon_{1}\left(F_{2}, F_{1}, E\right)\right\rangle= & {\left[\left\langle\epsilon_{1}\left(F_{2}, E\right)\right\rangle-\left\langle\epsilon_{1}\left(0_{3} E\right)\right\rangle\right] } \\
& -\left[\left\langle\epsilon_{1}\left(F_{1}, E\right)\right\rangle-\left\langle\epsilon_{1}(0, E)\right\rangle\right] \\
\equiv & \delta\left\langle\epsilon_{1}\left(F_{2}, E\right)\right\rangle-\delta\left\langle\epsilon_{1}\left(F_{1}, E\right)\right\rangle
\end{aligned}
$$

and

$$
\begin{aligned}
\delta\left\langle\epsilon_{2}\left(F_{2}, F_{1}, E\right)\right\rangle= & {\left[\left\langle\epsilon_{2}\left(F_{2}, E\right)\right\rangle-\left\langle\epsilon_{2}(0, E)\right\rangle\right] } \\
& -\left[\left\langle\epsilon_{2}\left(F_{1}, E\right)\right\rangle-\left\langle\epsilon_{2}(0, E)\right\rangle\right] \\
\equiv & \delta\left\langle\epsilon_{2}\left(F_{2}, E\right)\right\rangle-\delta\left\langle\epsilon_{2}\left(F_{1}, E\right)\right\rangle,
\end{aligned}
$$

where $\langle E(0, E)\rangle$ is the unperturbed pseudodielectric function of the sample. Equations (4a) and (4b) are the means by which $P E$ data will be analyzed, as next described.

\section{THEORETICAL BACKGROUND}

For an n-type GaAs surface exposed to air, it is known that, because of the existence of surface states and the adsorption of oxygen which is electronegative, the Fermi level is pinned at the surface, and that the energy bands are bent upward, giving rise to a consequent built-in field across the depletion region beneath the surface. ${ }^{30}$ Figure 2 shows the energy bands of an n-type GaAs surface with and without the effects of pump light irradiation. Under pump light irradiation, free carriers are generated within the sample. The carriers generated near the surface are separated by the built-in field to produce a photovoltage 
$\left(V_{p}\right)$, which in tarn reduces the band bending and the original field strength. This phenomenon is known as the photovoltaic effect.

Based on the depletion approximation, ${ }^{31,32}$ the built-in electric field strength due to the presence of a Schottky surface barrier in a uniformly doped semiconductor is known to vary linearly from a maximum value at the surface to zero at the edge of the depletion region, in the direction normal to the surface. Solving the Poisson's equation with the appropriate boundary conditions, the maximum built-in field strength $F_{\max }$ is ${ }^{31}$

$$
F_{\max }=\left\{\left[\left(2 e N_{D}\right) /\left(\epsilon_{0} \epsilon_{r}\right)\right]\left[V_{s}-\left(E_{C}-E_{F}\right) / e\right]\right\}^{1 / 2},
$$

where $e$ is the electron charge, $N_{D}$ is the doping density, $\epsilon_{0}$ is the dielectric constant of vacuum, $\epsilon_{r}$ is the relative dielectric constant of the sample material. $V_{s}=\left(E_{F, S}\right.$ $-k T) / e$ is the Schottky barrier height, where $E_{F, S}$ is the surface Fermi level and $k T$ is the thermal energy term. $E_{C}$ and $E_{F}$ are the conduction band minimum and the Fermi energy in the bulk, respectively.

In the low-field limit, Aspnes ${ }^{33}$ showed that the magnitude of the change in the dielectric function is directly proportional to the modulating voltage measured relative to the flatband condition, which implies that the following equation is valid for the situation shown in Fig. 2:

$$
\left|\delta\left\langle\epsilon_{2}\left(F_{2}, F_{1}, E\right)\right\rangle\right|=B V_{p},
$$

where $\delta\left\langle\epsilon_{2}\left(F_{2}, F_{1}, E\right)\right\rangle$ is given by $E q_{.}(3 b), B$ is a proportionality constant depending on the sample's properties only, and $V_{p}$ is the photovoltage.

Recently, Kanata et $a l^{6}{ }^{6}$ studied the surface Fermi level in GaAs (100), using PR and taking into account the photovoltaic effect at the semiconductor surface. They showed that, under the condition of $V_{s}-\left(E_{C}\right.$ $\left.-E_{F}\right) / e \gg V_{p}$, Eq. (6) also holds in the intermediate-field limit. According to Kanata et al., ${ }^{6}$ if the recombination current in the depletion region is neglected and the diffusion length of free carriers in the semiconductor is much longer than the penetration depth of the pump light, under the steady-state condition, the pump beam power density $P$, the Schottky barrier height $V_{s}$, and the photovoltage $V_{p}$ are related to each other through the following equation:

$$
\frac{P \gamma(1-R)}{h v}=\frac{A^{*} T^{2}}{e} \exp \left(-\frac{e V_{s}}{k T}\right)\left[\exp \left(\frac{e V_{p}}{\xi k T}\right)-1\right],
$$

where $\gamma \approx 1$ is the quantum efficiency in the sample, $h v$ is the photon energy of the pump light, $R$ is the refiectivity of the pump light at the sample surface, $\xi \approx 1$ is the ideality factor, and $A^{*}$ is the effective Richardson constant. For $n$-type GaAs with a free surface, $A^{*} \approx 8.0 \mathrm{~A}$ $\mathrm{cm}^{-2} \mathrm{~K}^{-2} \cdot 6,30,34$ Missous and Rhoderick ${ }^{35}$ showed that the value of $A^{*}$ was about $0.4 \mathrm{~A} \mathrm{~cm}-2 \mathrm{~K}^{-2}$ for epitaxially deposited Al on $n$-type GaAs, which was consistent with results found earlier by Srivastava et al. ${ }^{36}$ for $n$-type GaAs with contacts of $\mathrm{Al}$ and $\mathrm{Au}$. These results suggested that the Richardson constant might depend on the metal as well as on the semiconductor. However, in this work each sample was measured in as-received conditions, i.e., neither surface treatments nor metal contacts were applied to the sample. Thus, the choice of $A^{*} \approx 8.0 \mathrm{~A} \mathrm{~cm}^{-2} \mathrm{~K}^{-2}$ made here was based on the assumption that this value would hold for $n$-type GaAs with a free surface, ${ }^{6,30,34}$ Our results for $V_{s}$ are not very sensitive to $A^{*} ; V_{s}$ values obtained assuming the lower $A^{*}$ value are only about $0.05 \mathrm{~V}$ below those obtained assuming the higher $A *$ value.

Solving for $V_{p}$ from Eq. (7), one obtains the following expression:

$$
V_{p}=\frac{\xi k T}{e} \ln \left(\frac{\operatorname{Pr}(1-R)}{\left(A^{*} T^{2} / e\right) h v \exp \left[-\left[e V_{S} /(k T)\right]\right.}+1\right) .
$$

From Eqs. (6) and (8), the following equation can be obtained:

$$
\begin{aligned}
& \left|\delta\left\langle\epsilon_{2}\left(F_{2}, F_{1}, E\right)\right\rangle\right| \\
& \equiv\left|\delta\left\langle\epsilon_{2}\right\rangle\right|=B \frac{\xi k T}{e} \\
& \quad \times \ln \left(\frac{P \gamma(1-R)}{\left(A^{*} T^{2} / e\right) h v \exp \left[-e^{V} /(k T)\right]}+1\right),
\end{aligned}
$$

which directly relates the experimentally measured parameters $\delta\left\langle\epsilon_{2}\right\rangle, P$, and $R$ to the unknown parameter $V_{s}$ at a given temperature.

Thus, if two different pump beam power densities, nameiy, $P_{1}$ and $P_{2}$, are used, two corresponding $\delta\left\langle\epsilon_{2}\right\rangle_{1}$ and $\delta\left\langle\epsilon_{2}\right\rangle_{2}$ can be measured using $\mathrm{PE}$, which means that, from Eq. (9), one has

$$
\begin{aligned}
\left|\delta\left\langle\epsilon_{2}\right\rangle_{i}\right|= & B \frac{\xi k T}{e} \\
& \times \operatorname{in}\left(\frac{p_{i} \gamma(1-R)}{\left(A^{*} T^{2} / e\right) h v \exp \left[-e V_{s} /(k T)\right]}+1\right) \\
& i=1,2 .
\end{aligned}
$$

From Eq. (10), one has

$$
\begin{aligned}
\frac{\mid \delta\left\langle\epsilon_{2}\right\rangle_{1}}{\left|\delta\left\langle\epsilon_{2}\right\rangle_{2}\right|} \\
=\ln \left(\frac{P_{1} \gamma(1-R)}{\left(A^{*} T^{2} / e\right) h v \exp \left[-e V_{s} /(k T)\right]}+1\right) \\
\quad \times\left[\ln \left(\frac{P_{2} \gamma(1-R)}{\left(A^{*} T^{2} / e\right) h v \exp \left[-e V_{s} /(k T)\right]}+1\right)\right]^{-1} .
\end{aligned}
$$

Equation (11) can be rewritten in the following form: 
TABLE I. Measured and calculated data at $E=1.425 \mathrm{eV}$ for both samples.

\begin{tabular}{|c|c|c|c|c|c|c|c|}
\hline$N_{D}\left(\mathrm{~cm}^{-3}\right)$ & $\left|\delta\left\langle\epsilon_{2}\right\rangle_{1}\right|$ & $\left|\delta\left\langle\epsilon_{2}\right\rangle_{2}\right|$ & $P_{1}\left(\mathrm{~mW} / \mathrm{cm}^{2}\right)$ & $P_{2}\left(\mathrm{~mW} / \mathrm{cm}^{2}\right)$ & $R$ & $V_{s}(\mathrm{~V})$ & $E_{F_{s}, S}(\mathrm{eV})$ \\
\hline $1.3 \times 10^{17}$ & $0.057 \pm 0.002$ & $0.046 \pm 0.002$ & 7.73 & 0.58 & 0.344 & $0.84 \pm 0.06$ & $0.84 \pm 0.06$ \\
\hline $4.0 \times 10^{15}$ & $0.060 \pm 0.002$ & $0.038 \div 0.0022$ & 7.89 & 0.21 & 0.344 & $0.69 \pm 0.06$ & $0.69 \pm 0.06$ \\
\hline
\end{tabular}

$$
\begin{aligned}
& {\left[\frac{P_{1} \gamma(1-R)}{\left(A^{*} T^{2} / e\right) h v \exp \left[-e V_{S} /(k T)\right]}+1\right]\left|\delta\left\langle\epsilon_{2}\right\rangle_{2}\right|} \\
& =\left[\frac{p_{2} \gamma(1-R)}{\left(A^{*} T^{2} / e\right) h v \exp \left[-e V_{s} /(k T)\right]}+1\right]\left|\delta\left\langle\epsilon_{2}\right\rangle_{1}\right| .
\end{aligned}
$$

Equation (12) can be solved graphically to give the Schottky barrier height $V_{s}$ and consequently the surface Fermi level $E_{F, S}$ from $V_{s}=\left(E_{F, S}-k T\right) / e$.

For a uniform electric field $F$, the field-induced change in the dielectric function $\epsilon$ from the Franz-Keldysh effect, near an $M_{0} C P$ (e.g., near the GaAs band-gap $E_{0} C P$ ), is given by ${ }^{27,33}$

$$
\begin{aligned}
\delta \epsilon(F, E) & =\delta \epsilon_{1}(F, E)+i \delta \epsilon_{2}(F, E) \\
& \equiv\left(C \theta^{1 / 2} / E^{2}\right)[G(\eta)+i F(\eta)],
\end{aligned}
$$

where parameter $C$ contains the interband optical transition matrix element. Parameters $\theta$ and $\eta$ are defined by $\left(e^{2} F^{2} / 2 \mu h\right)^{1 / 3}$ and $\left(E_{0}-E\right) / h \theta$, respectively, where $\mu$ is the interband reduced effective mass at the $M_{0} \mathrm{CP}$ in the direction of the field. $G(\eta)$ and $F(\eta)$ are the electro-optic functions, given by combinations of Airy functions, their derivatives, and a unit step function. Note that Eq. (13) does not include broadening.

In the case of a nomuniform field $F(z)$ where its value varies spatially over a depth $d$ in the $z$ direction normal to the surface, the overall effective change in the dielectric function can be approximated by 27,33

$$
\delta \varepsilon(F, E)=-2 i K \int_{-d}^{0} \delta \epsilon[F(z), E] \exp (-2 i K z) d z
$$

where $\delta[F(z), E]$, the change due to a local field, is obtained from $E q$. (13) and $K=2 \pi \epsilon^{1 / 2} / \lambda$ is the unperturbed propagation constant of the probe light in the sample. Note that $\mathrm{EG}$. (14) was derived assuming that the probe beam was incident normal to the surface.

Details about the treatments of broadening have been given in Ref. 27 . In this work, the effect of broadening was assumed to be Lorentzian type. Consequently, the fieldinduced change in $\epsilon$ in the presence of Lorentzian broadening was obtained from the unbroadened term $\delta \in(F, E)$ by using ${ }^{37}$

$$
\delta \epsilon(F, E+i \Gamma)=\frac{1}{\pi} \int_{0}^{\infty} \frac{\delta \epsilon\left(F_{0}, E^{\prime}\right) \Gamma}{\left(E-E^{\prime}\right)^{2}+\Gamma^{2}} d E^{\prime}
$$

where $\Gamma$ is the broadening parameter.

Recently, Batchelor et al. ${ }^{38}$ made extensive ER studies in GaAs. They modeled the effect of broadening in the $E_{0} C P$ region by expressing $\Gamma$ in terms of
$\Gamma_{0} \cdot \exp \left[\delta \cdot\left(E^{\prime}-E_{0}\right)\right]$, where $\Gamma_{0}$ is the broadening parameter and $\delta$ is a proportionality factor. This empirical expression was proposed based on the assumption that more highly excited electrons in the conduction band would undergo more rapid relaxation due to collisions with lattice defects, and therefore $\Gamma$ would be likely to increase with increasing energy. This approach was also adopted in this work.

\section{$V$ RESULTS AND DISCUSSION}

To determine the surface Fermi level, $P E$ measurements were first taken with two different punp beam power densities at a particular photon energy in the above-band-gap $E_{0}$ region, where the field-induced change in $\langle\epsilon\rangle$ was large. Using Eq. (12), the measured datas i.e., $\delta\left\langle\epsilon_{2}\right\rangle_{1}$ and $\delta\left\langle\epsilon_{2}\right\rangle_{2}$, were analyzed graphically to give the Schottky barrier height $V_{s}$. Table I lists the calculated values of Schottky barrier height $V_{s}$ and surface Fermi level $E_{F, S}$ for the two GaAs samples, together with the experimentally measured data, namely, $\left|\delta\left\langle\epsilon_{2}\right\rangle_{1}\right|,\left|\delta\left\langle\epsilon_{2}\right\rangle_{2}\right|$, the two corresponding pump beam power densities $P_{1}$ and $P_{2}$, the probe light photon energy $\bar{E}$ at which the measurements were made, and the reflectivity $R$ at the wavelength of $632.8 \mathrm{~nm}$. As can be seen, the values of surface Fermi level $E_{F, S}$ in both samples are within the range between 0.6 and $1.0 \mathrm{eV}$, comparable with those determined by $\mathrm{PR}^{4,34}$ high resolution electron energy loss spectroscopy ${ }^{39}$ and photoemission spectroscopy. ${ }^{40}$

Next, $\delta\langle\varepsilon\rangle$ measurements were made with a single pump beam power density, over a spectral range from 1.30 to $1.65 \mathrm{eV}$ near the $E_{0} C P$ region. Figures 3 and 4 show the experimental data and theoretical calculations for the two samples studied. These experimental results represent the direct observation of the Franz-Keldysh effect, without using the Kramers-Kronig transiormation. The theoretical calculations were obtained using the following procedure: the unbroadened results were first obtained using Eq. (14) together with Eq. (13), where only the heavy-hole contributions were considered (the reduced mass $\mu$ was taken to be $0.059 m_{e}$, where $m_{e}$ is the free electron mass) and the unperturbed propagation constant $K$ for GaAs was calculated using the data from Ref. 41 . These unbroadened results were then broadened using Eq. (15) to give the final theoretical data. For all the calculations made here, a linearly varying field profile was assumed with a maximum built-in field $F_{\max }$ at the surface and a minimum field $F_{\min }$ at the edge of the depletion depth $d$. Parameters, such as $F_{\max }, d, \Gamma_{0}, \delta$, and the $\mathrm{CP}$ energy $E_{0}$, were variables to be determined in the theoretical calculations, while $F_{\min }$ was held fixed at $3.0 \times 10^{2} \mathrm{~V} / \mathrm{cm}$, a value small enough to make significant contributions to the overall theoretical spectra. 

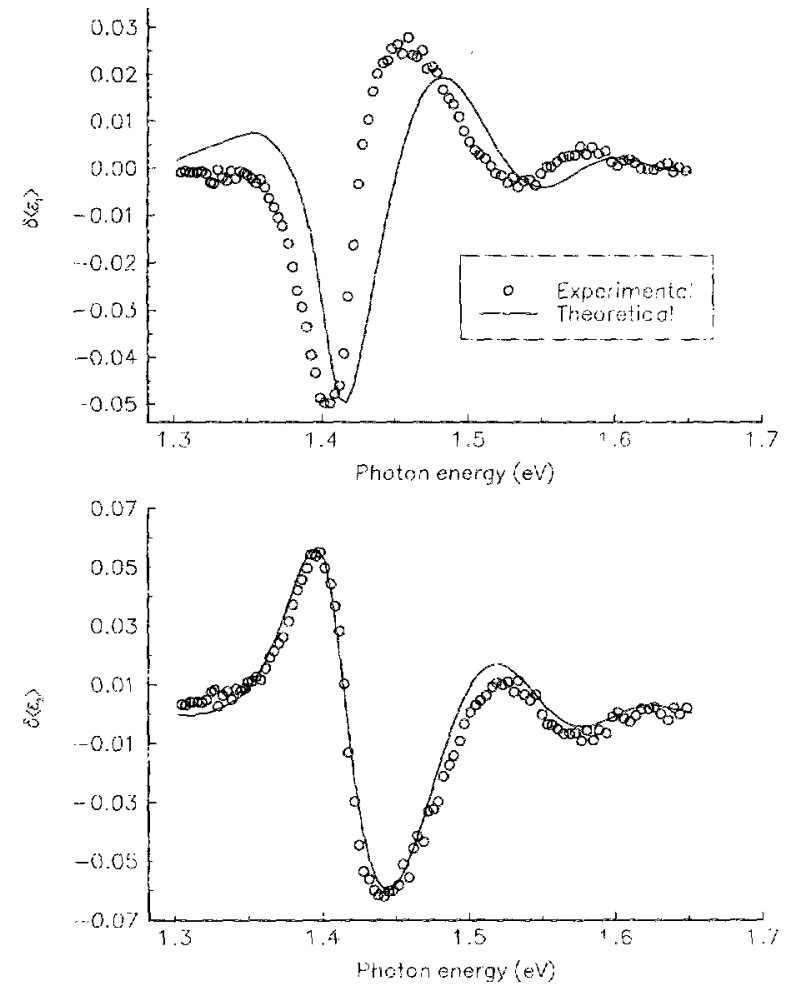

FIG. 3. Experimental and theoretical data in the $E_{0} C P$ region, for the GaAs sample with $N_{D} \approx 1.3 \times 10^{17} \mathrm{~cm}^{-3}$. The pump beam power density was $6.85 \mathrm{~mW} / \mathrm{cm}^{2}$.

More specifically, for the sample with the doping density $N_{D} \approx 1.3 \times 10^{17} \mathrm{~cm}^{-3}$, the theoretical results shown by the solid lines in Fig. 3 were generated using a field $F_{\max }$ of $1.63 \times 10^{5} \mathrm{~V} / \mathrm{cm}$, a depth $d$ of $91.0 \mathrm{~nm}$, a $C P$ energy $E_{0}$ of $1.41 \mathrm{eV}$, and a broadening parameter $\Gamma_{0}=0.015 \mathrm{eV}$ with $\delta=2.0 \quad \mathrm{eV}^{-1}$. Using $F_{\max }=1.63 \times 10^{5} \quad \mathrm{~V} / \mathrm{cm}$, $N_{D} \approx 1.3 \times 10^{17} \mathrm{~cm}^{-3}$, and Eq. (5), along with the expression $V_{s}=\left(E_{F, S}-k T\right) / e$, the surface Fermi level $E_{F, S}$ was found to be $\sim 0.77 \mathrm{eV}$, which compares well with the corresponding value listed in Table $\mathbb{1}$. For the sample with $N_{D} \approx 4.0 \times 10^{17} \mathrm{~cm}^{-3}$, the theoretical calculations in Fig. 4 were obtained with a field $F_{\max }$ of $2.71 \times 10^{5} \mathrm{~V} / \mathrm{cm}$, a depth $d$ of $49.0 \mathrm{~nm}$, a $C P$ energy $E_{0}$ of $1.41 \mathrm{eV}$, and a broadening parameter $\Gamma_{0}=0.022 \mathrm{eV}$ with $\delta=3.0 \mathrm{eV}^{-1}$. Using $F_{\max }=2.71 \times 10^{5} \mathrm{~V} / \mathrm{cm}, N_{D} \approx 4.0 \times 10^{17} \mathrm{~cm}^{-3}$, the surface Fermi level $E_{F, S}$ was calculated to be $\sim 0.65 \mathrm{eV}$, which again compares well with the corresponding value listed in Table I. In addition, it can be seen that, as the doping level increases, so does the broadening, likely due to the increase in collisions between electrons and lattice defects.

It should be mentioned that the above field profile was chosen based on the depletion approximation ${ }^{31,32}$ for a bulk semiconductor of uniform doping. In other words, it was assumed that the experimental PE spectra measured in this work were due to the Franz-Keldysh effect on the dielectric function of the sample produced by a linearly varying field over a depletion depth $d$. Therefore, the model also assumed a linear field profile, and the modeled (theoretical) spectra from Eq. (14) contained contributions from
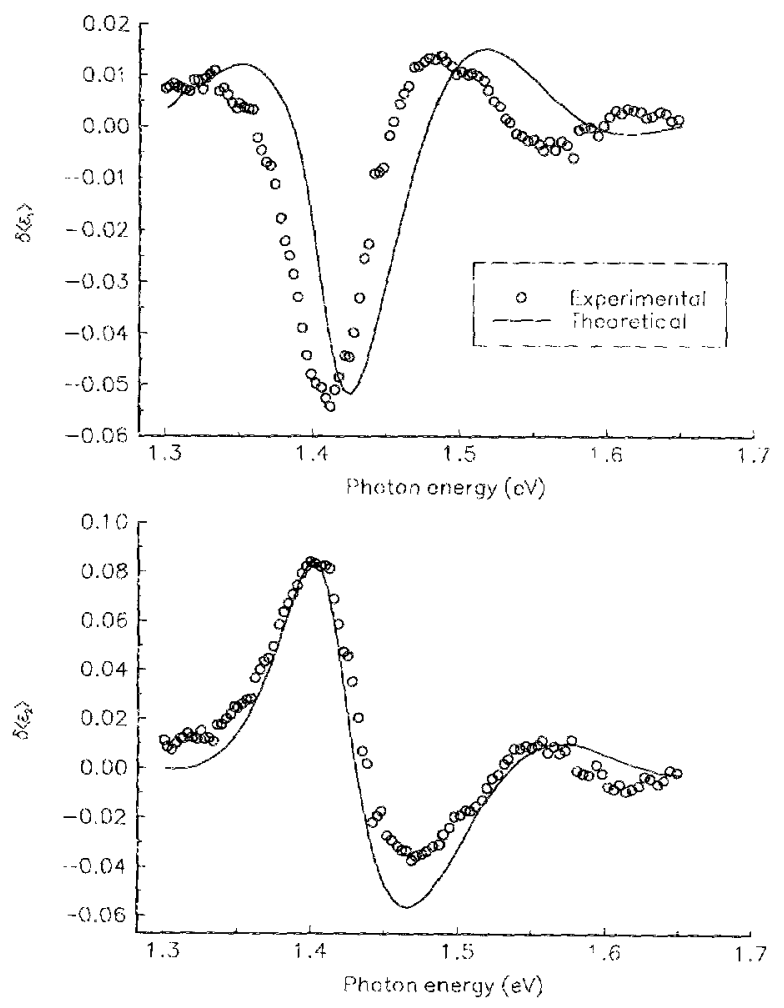

FIG. 4. Experimental and theoretical data in the $E_{0} C P$ region, for the GaAs sample with $N_{D} \approx 4.0 \times 10^{17} \mathrm{~cm}^{-3}$. The pump beam power density was $5.60 \mathrm{~mW} / \mathrm{cm}^{2}$.

the full profile, rather than from the maximum built-in surface field alone or from the average field.

Observing the $\delta\left\langle\epsilon_{1}\right\rangle$ spectra in Figs. 3 and 4 , it can be seen that each theoretical curve has a similar type of shift towards higher energies with respect to experimental curve. The cause of this type of shift is believed to be mainly due to the facts that the effects of the native oxide overlayer were not included in the theory, and that the theoretical calculations were made assuming a normal incidence for the probe beam rather than $75^{\circ}$, the actual probe beam angle of incidence for the measured data. Note that, for $n$-type GaAs, $\delta\left\langle\epsilon_{1}\right\rangle$ and $\delta\left\langle\epsilon_{2}\right\rangle$ are approximately proportional to $\delta \Delta$ and $\delta \psi$, respectively, in the $E_{0} \mathrm{CP}$ region as shown in Figs. 5 and $6 . \delta \Delta$, being related to the phase change, ${ }^{29}$ is extremely sensitive to overlayers (such as a native oxide) and changes in the angle of incidence, this is why the largest discrepancies between the experiment and theory are seen from the $\delta\left\langle\epsilon_{1}\right\rangle$ spectra in Figs. 3 and 4 . On the other hand, $\delta \psi$, being related to the amplitude change, ${ }^{29}$ is less sensitive to overlayers, and this is why good agreement between the experiment and theory has been obtained in the $\delta\left\langle\epsilon_{2}\right\rangle$ spectra. This observation also illustrates the importance of having the experimental $\delta\left\langle\epsilon_{1}\right\rangle$ spectrum available in the data analysis. Quantitative analysis of these overlayer and non-normal incidence effects produces good fits to the $\delta\left\langle\epsilon_{1}\right\rangle$ spectra. ${ }^{42}$

\section{SUMMARY}

Photoellipsometry, a new contactless optical method, has been described. It allows direct measurements of the 

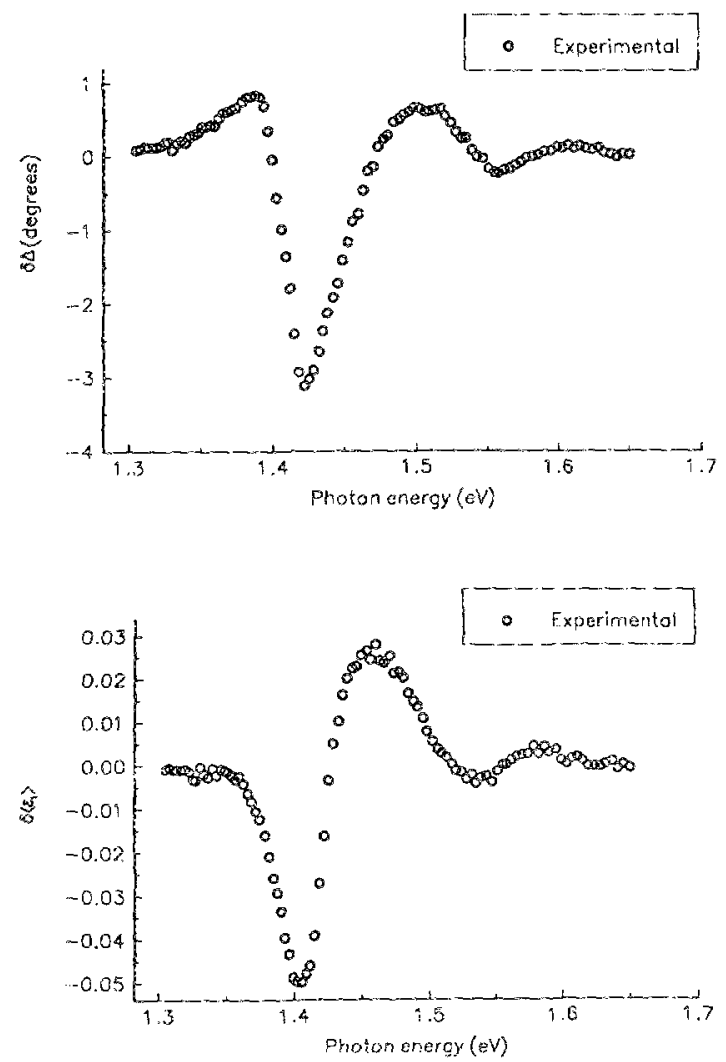

FIG. 5. Experimentally measured $\delta\left(\epsilon_{1}\right)$ and $\delta \Delta$ spectra, for the GaAs sample with $N_{D} \approx 1.3 \times 10^{17} \mathrm{~cm}^{-3}$. Note that $\delta\left\langle\epsilon_{1}\right)$ is approximately proportional to $\delta \Delta$.
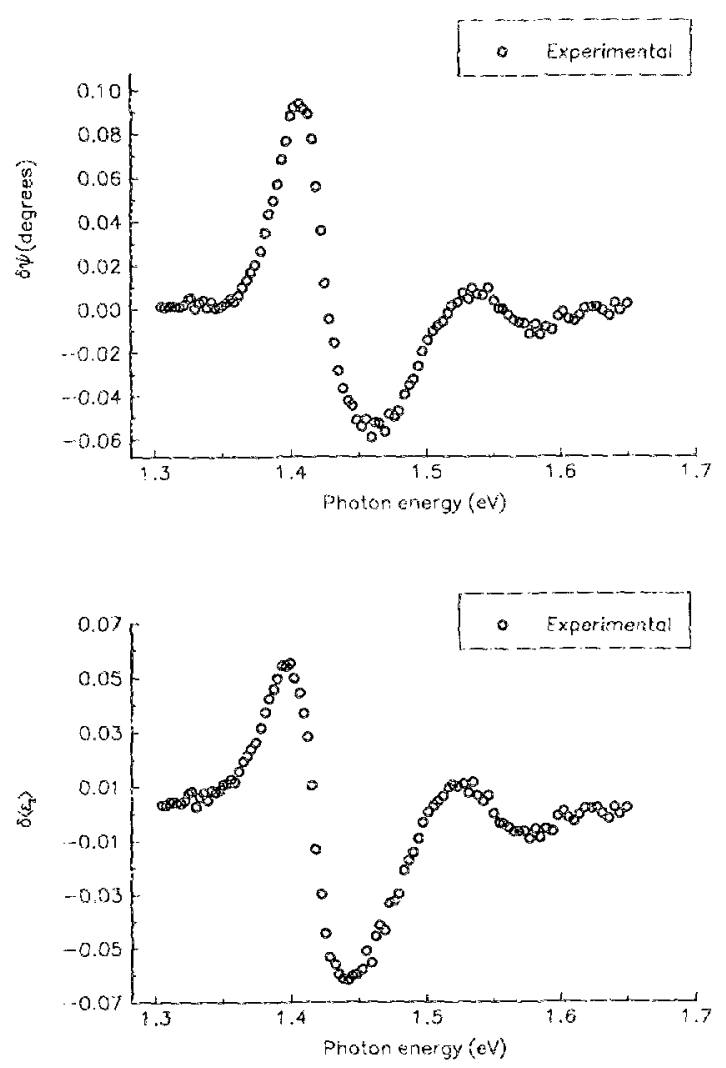

FiG. 6. Experimentally measured $\delta\left\langle\epsilon_{2}\right\rangle$ and $\delta \psi$ spectra, for the GaAs sample with $N_{D} \approx 1.3 \times 10^{17} \mathrm{~cm}^{-3}$. Note that $\delta\left\langle\epsilon_{2}\right\rangle$ is approximately proportional to $\delta \psi$. built-in field-induced changes in both the real and imaginary parts of the pseudodielectric function of a semiconductor under study, without the need for a KramersKronig transformation. The method was used to determine the surface Fermi level $E_{F, S}$ in two $n$-type GaAs (100) samples. The measured data were analyzed in terms of the pump beam power density and the probe beam photon energy. Both techniques have yielded consistent results for $E_{F, S}$.

'B. O. Seraphin, in Semiconductors and Semimetals, edited by R. K. Willardson and A. C. Beer (Academic, New York, 1972), Yol. 9, p. 1

${ }^{2}$ E. Y. Wang and W. A. Albers, Phys. Lett. A 27, 347 (1968).

${ }^{3}$ F. H. Pollak and H. Shen, J. Electron. Mater. 19, 399 (1990).

${ }^{4}$ D. K. Gaskill, N. Botkka, and R. S. Sillmon, J. Vac. Sci. Technol B 6, $1497(1988)$

'C. Van Hoof, K. Deneffe, J. D. Boeck, D. J. Arent, and O. Borgh, Appl. Phys. Lett. 54, 608 (1989).

${ }^{6}$ T. Kanata, M. Matsunaga, H. Takakura, and X. Hamakawa, J. Appi. Phys. 68,5309 (1990).

${ }^{7}$ H. Shen, S. H. Pan, Z. Hang, J. Lang, F. H. Pollak, J. M. Woodall, and R. N. Sacks, Appl. Phys. Lett. 53, 1080 (1988).

${ }^{8}$ B. D. Cahan and R. F. Spanier, Surf. Sci. 16, 166 (1969)

${ }^{9}$ D. E. Aspnes and A. A. Studna, Appl. Opt. 14, 220 (1975),

10. D. Aspnes and A. A. Studna, Phys. Rev. B 27, 985 (1983).

"L. Vinä and M. Cardona, Phys. Rev. B 29, 6739 (1984).

${ }^{12}$ D. E. Aspnes, S. M. Kelso, R. A. Logan, and R. Bhat, J. Appl. Phys. 60,754 (1986).

${ }^{13}$ P. Lautenschlager, M. Garriga, S. Logothetidis, and M. Cardona, Phys. Rev. B 35, 9176 (1987).

${ }^{14}$ Y. G. Snyder and Y. M. Xiong, Surf. Interiace Anal. 18, 107 (1992).

i5. E. Aspnes, I. B. Theeten, and F. Hottier, Phys. Rev. B 20, 3292 (1979).

${ }^{16}$ M. Erman, J. B. Theeten, N. Vodjdani, and Y. Dernay, J. Vac. Sci. Technol. B 1, 328 (1983).

${ }^{17}$ K. Vedam, P. J. McMarr, and J. Narayan, Appl. Phys. Lett. 47, 339 (1985).

${ }^{18}$ Y. M. Xiong and P. G. Snyder, Thin Solid Films 220, 303 (1992).

${ }^{19}$ P. G. Snyder, M. C. Rost, G. H. Bu-Abbud, J. A. Woollam, and S. A. Alterowitz, J. Appi. Phys. 60, 3293 (1986).

${ }^{20}$ J. A. Woollam, P. G. Snyder, A. W. McCornick, A. K. Rai, D. Ingram, and P. P. Pronko, J. Appl. Phys. 62, 4867 (1987).

${ }^{21}$ S. A. Alterovitz, J. A. Woollam, and P. G. Snyder, Sold State Technol. 31, $99(1988)$

${ }^{22}$ N. V. Nguyen and K. Vedam, J. Appl. Phys. 67, 3555 (1990).

${ }^{23}$ A. B. Buckman and N. M. Bashara, Phys Rev, 174, 719 (1968).

${ }^{24}$ S. Tachi, A. Moritani, and J. Nakai, J. Appl. Phys. 50, 5461 (1979).

${ }^{25}$ O. J. Glembocki, Proc. SPIE 452, 130 (1983).

${ }^{26}$ P. G. Snyder, J. E. Oh, J. A. Woollam, and R. E. Owens, Appl. Phys. Lett. 51, $770(1987)$.

${ }^{27}$ D. E. Aspnes and N. Bottka, in Ref. 1, p. 457.

${ }^{29}$ I. I. Pankove, Optical Processes in Semiconductors (Prentice-Hall, New Jersey, 197!), Chap. 14, p. 302.

${ }^{29}$ R. M. A. Azzam and N. M. Bashara, Ellipsometry and Polarized light (North-Holland, Amsterdam, 1977).

${ }^{30} \mathrm{G}$. Y. Robinson, in Physics and Chemistry of IIT-V Compound Semiconductor Interfoces, edited by C. W. Wilmsen (Plenum, New York, 1985), Chap. 2, p. 73.

${ }^{31}$ E. H. Rhoderick and R. H. Williams, Metal-Semiconductor Contacts, 2nd ed. (Clarendon, Oxford, 1988).

${ }^{32}$ J. L. Shay, Phys. Rev. B 2, 803 (1970).

${ }^{33}$ D. E. Aspnes, in Handbook of Semiconductors, edited by T. S. Moss (North-Holland, Amsterdam, 1980), Vol. 2, p. 109.

${ }^{34} \mathrm{X}$. Yin, H.-M. Chen, F. H. Pollak, Y. Chan, P. A. Monotano, P. D. Kirchner, G. D. Pettit, and J. M. Woodall, J. Vac. Sci. Technol. A 10, 131 (1992).

${ }^{35}$ M. Missous and E. H. Rhoderick, J. Appl. Phys. 69, 7142 (1991).

${ }^{36}$ A. K. Srivastava, B. M. Arora, and S. Guha, Solid-State Electron. 24 , $185(1981)$. 
${ }^{37}$ M. Cardona, Modulation Spectroscopy (Academic, New York, 1969), Chap. 7, p. 182.

${ }^{38}$ R. A. Batchelor, A. C. Brown, and A. Hamnet, Phys. Rev. B 41 , 1401 (1990).

${ }^{39}$ Y. Chen, S. Nannarone, J. Schaefer, I. C. Hermanson, and G. I. Lapeyre, Phys. Rev. B 39, 7653 (1989).
${ }^{40}$ W. E. Spicer, I. Lindaw, P. S. Keath, C. Y. Su, and P. Chye, Phys. Rev. Lett. 44, $420(1980)$.

${ }^{41}$ E. D. Palik, in Handbook of Optical Constants of Solids, edited by E. D. Palik (Academic, New York, 1985), p. 429.

${ }^{42}$ Y. M. Xiong, Ph.D. dissertation. 